\title{
Utilización por cuenta propia de los Servicios de Urgencias Hospitalarias: razones que dan las personas con problemas de salud de baja complejidad para utilizar estos servicios
}

\author{
Non-Urgent and Non-Referred Patients Presenting \\ to the Emergency Department (ED): Understanding \\ the Reasons of the Non Urgent Patients to Visit the ED
}

\author{
Manuel EsPinel VALLeJo \\ Departamento de Sociología I, Facultad de Ciencias Políticas y Sociología, \\ Universidad Complutense de Madrid \\ mespinel@cps.ucm.es \\ Marcial RoMero LóPEz \\ Departamento de Sociología I, Facultad de Ciencias Políticas y Sociología, \\ Universidad Complutense de Madrid \\ marcialromero@cps.ucm.es \\ Lorenzo Fernández Franco \\ Universidad Complutense de Madrid \\ ferfran@estad.ucm.es \\ Juan TORRES MACHO \\ Hospital Infanta cristina, Parla Madrid \\ juantorresm@salud.madrid.org \\ Sergio D'Antonio MaCeiras \\ Universidad Complutense de Madrid \\ sergiodantonio@gmail.com
}

Recibido: 25.10.2010

Aprobado definitivamente: 15.11.2010

\section{RESUMEN}

Objetivos: Los objetivos de este estudio son: 1) Comprender las razones y los motivos que impulsan a las personas con problemas de salud de baja complejidad (PSBC) para acudir, por cuenta propia y sin ser remitidos por su médico de cabecera (MC), al servicio de urgencias de un hospital general (SUH). 2) Conocer la percepción sobre el grado de gravedad y preocupación atribuidos por las personas a su problema de salud. 3) Determinar los factores que influyen en la decisión de acudir por cuenta propia al SUH. Metodología. Diseño: Estudio transversal a través de entrevista personal con cuestionario estructurado, mediante muestreo no probabilístico por cuotas. Contexto: SUH del Hospital Infanta Cristina de Parla, Madrid, España. Participantes: Se entrevistaron a pacientes seleccionados por los niveles IV y V de la Escala Española de Triaje, que acudieron por cuenta propia al SUH, de lunes a domingo, entre las 10:00 
y las 22:00 horas, desde el 26 de abril al 30 de Julio de 2010. Principales aspectos considerados: Características socio económicas; experiencia previa con su centro de salud (CS) y con el SUH; gravedad y preocupación percibidos en relación con el problema de salud actual; utilización previa de asistencia sanitaria en relación con su problema actual de salud; razones para la utilización del SUH en relación con su problema actual de salud, evaluación comparativa entre el SUH y el CS.

Resultados: Se entrevistaron a 492 pacientes de 8.558 elegibles. El 73\% acudió directamente a las urgencias sin consultar al MC. El 44\% consideró que el SUH contaba con mejores medios técnicos para resolver el problema de salud, el $35 \%$ porque a esa hora ya no les atendían en el CS, el $22 \%$ porque le resolvía más rápidamente el problema de salud y el $20 \%$ acudió por comodidad. El $27 \%$ restante había acudido previamente al MC y posteriormente decidió acudir a urgencias porque no mejoró con el tratamiento que le prescribió el MC (71\%) y porque no estaba satisfecho con el tratamiento y quería una segunda opinión $(39 \%)$. El 70\% consideró que su problema de salud no era grave pero pensaba que sí era merecedor de atención urgente o rápida. El 63\% expresó alta preocupación por su problema. El 63\% consideró que el actual problema de salud no podía ser resuelto por su MC en caso de ser atendido en ese momento. Finalmente el 50\% consideró que el SUH tenía más capacidad para resolver los problemas de salud que el CS. Conclusión: La mejor eficacia y efectividad, en términos de uso y gestión de recursos técnicos y del tiempo para resolver el problema de salud, y la comodidad, en términos de horarios, disponibilidad y accesibilidad, parecen ser las principales razones por las cuales las personas con problemas de salud de baja complejidad utilizan por cuenta propia los SUH. La preocupación, más que la gravedad, parece movilizar activamente la búsqueda de solución al problema de salud de manera rápida y urgente. Estas razones parecen estar condicionadas en la experiencia institucional previa con el CS..

Palabras Clave: Utilización de los servicio de urgencias hospitalarias, problemas de salud de baja complejidad, razones, motivos y factores asociados

\section{ABSTRACT}

Objectives: The objectives of this study are: 1) To understand the reasons of non-urgent non-referred patients to use the Emergency Department (ED), 2) To understand the "urgency" and "concern" perceptions of non-urgent non-referring patients. 3) To determinate the associated factors with the use the ED for non-urgent non-referred patients.

Methods: Design: Descriptive cross-sectional study. Setting: Emergency Department (ED) of "Infanta Cristina" General Hospital, Parla, Madrid, Spain. Patients: non-referred non-urgent adult patients were selected by Emergency Department Triage system in levels IV and V, on weekdays between 10:00 and 22:00 hours, from April 26 to July 30, 2010, to be surveyed using a structured face-to-face interview. Main outcome measures: sociodemographic characteristics, personal experience with the health care centre (HCC) and ED, urgency and concern of symptoms, previous health care use regarding current health problem, reasons to visit the ED, comparative evaluation of ED and HCC.

Results: $73 \%$ of the patients came directly to the ED. More efficient technical services (42\%), the HCC was closed in this time (35\%), promptly resolution of the health problem $(22 \%)$ and more accessibility $(20 \%)$ were the most important reasons to come directly to ED. $27 \%$ of the patients went previously to the HCC and then they came to the ED because they did not get better with the treatment prescribed (71\%) and they went to know a second opinion (39\%). $70 \%$ of the patients considered that their health problem was not serious but they thought this situation had to be resolved promptly. $63 \%$ of the patients were very worried for their health problem and the same proportion considered that their family doctor could not resolve this problem. Finally, 50\% considered that the ED had more technical capacity to resolve their health problem.

Conclusions: High efficacy and effectiveness, in terms of technical resources and promptness, were the main reasons of non-referred non-urgent adult patients to use the ED. The high preoccupation, but not the perception of seriousness perception, of the health problem was the most important factor to seek care in an active and quickly way. The previous institutional experience in HCC could influence those reasons.

KEYwORDS: Emergency Department utilization, non urgent use, understanding reasons and associated factors.

\section{SUMARIO}

1. Introducción. 2. Metodología. 3. Resultados. 4. Discusión. 5. Conclusiones. 6. Bibliografía. 


\section{INTRODUCCIÓN}

En los últimos 20 o 30 años los Servicios de Urgencias Hospitalarios (SUH) se han convertido en una pieza clave de los sistemas de salud, particularmente de la red de asistencia sanitaria, en las sociedades modernas. Realizan un servicio público especialmente relevante, 24 horas al día durante los 365 días del año, independientemente de la condición socioeconómica del paciente. La labor de los SUH no sólo se ha concentrado en la atención inmediata de pacientes críticamente enfermos o severamente lesionados, sino que se ha extendido a otras áreas como la vigilancia en salud pública, atención de desastres o de atentados terroristas, realización de procedimientos (transfusiones de sangre), etc. (Derlet, Richards, Kravitz, 2001; Richardson, Hwang, 2001a y 2001b; Weiss, et al, 2004; Sánchez, Cabanillas, 2005; Moskop, et al, 2009a). En España La Ley General de Sanidad garantiza la utilización de los SUH de forma libre, gratuita y universal.

En relación con la utilización de los $\mathrm{SUH}$, existe un consenso más o menos generalizado entre los expertos en el área de urgencias y emergencias, según el cual, el número de consultas que realizan los SUH se ha incrementado de manera significativa en todos los países desarrollados en los últimos años (Alonso, et al, 1993; Sempere-Selva, T., et al, 2001; Richardson, Hwang, 2001a; Richardson, Hwang, 2001b; Sempere-Selva, T., et al 2001; Richardson, et al, 2002; Hwang, Concato, 2004; Ragin, D., et al, 2005; Pasarin, M., et al, 2006; Zaragoza, 2009; Tranquada, et al, 2010). En el caso de Estados Unidos, por ejemplo, según The National Hospital Ambulatory Medical Care Survey de 2006, el total de urgencias atendidas aumentó de 90,3 millones en 1996 a 119,2 en 2006. Esto significa un incremento de 2,9 millones de consultas $(3,3 \%)$ por año (US Department of Health and Human Services, 2008). En el caso de Inglaterra, el número de urgencias atendidas pasó de 9,2 millones en 1979 a 11,2 millones en 1990, es decir cerca de 200 mil (2\%) por año. Ahora bien, entre 1990 y 2007 el número de urgencias atendidas pasó de 11,2 a 12,3 millones, es decir un incremento de $64 \mathrm{mil}$ urgencias (menos de 1\%) por año (Murphy, 1998; National Health System, 2009). En Espa- ña, según La Estadística de Establecimientos Sanitarios con Régimen de Internado, el total de urgencias atendidas aumentó de 17,9 millones en 1997 a 25,3 millones en 2006. Esto significa un incremento de cerca de 1 millón de consultas por año, es decir, $4,6 \%$ anual.

También existe un consenso general entre los expertos según el cual este incremento de la utilización de los SUH está asociado a un importante aumento de la carga o presión asistencial de estos servicios, particularmente en los últimos 20 años, con su consecuente saturación (Grumbach, Keane, Bindman, 1993; Sempere, et al, 1999; Derlet, Richards, 2000; Derlet, Richards, Kravitz, 2001; Schneider, et al, 2001; Richardson, Asplin, Lowe, 2002;; Schneider, et, al, 2003; Bragulat, E., et al, 2003; Fatovich, Hirsch, 2003; Trzeciak, Rivers, 2003; Hwang, Concato, 2004; Weiss, et al, 2004; American College of Emergency Physicians, 2008; Hoot, Aronsky, 2008; Moskop, et al, 2009a y 2009b; Bernstein, S., et, al, 2009; Stead, Jain, Decker, 2009). Este fenómeno tampoco es ajeno en los servicios de urgencias de los hospitales españoles (Sánchez, et al, 2003; Zaragoza, et al, 2009). Por otra parte, esta saturación de los SUH no sólo forma parte de la literatura especializada sobre este tema (Richardson, Hwang, 2001a), sino también es fuente de debate público en los medios de comunicación por su impacto sobre la calidad de la asistencia sanitaria, como es el caso de España (Pérez, 2007; Sánchez, Ortiz, 2007). Es importante no perder de vista que, en última instancia, la saturación de los servicios de urgencia compromete, en mayor o menor medida, la atención de los pacientes seriamente enfermos especialmente porque genera retrasos en los diversas etapas del proceso de atención y porque aumentan los errores en las decisiones que se toman y en los procedimientos que se realizan en cada una de estas etapas (Bernstein et al, 2009)

Una compleja red de factores puede contribuir a la saturación de los SUH: a) Aumento del número absoluto de pacientes que utilizan los SUH; b) Aumento de la utilización de los SUH, tanto por pacientes con problemas de salud de baja complejidad (PSBC) como, por el contrario, por pacientes con problemas de salud de alta complejidad; c) Disminución absoluta o relativa de las camas tanto en el SUH como en el propio hospital, en relación con la demanda del servicio 
de urgencias; d) Criterios contrapuestos relacionados con la gravedad del los pacientes entre el SUH y el Servicio de Atención Primaria; e) Mejora de la capacidad resolutiva de los SUH que permite observar y tratar pacientes que antes eran hospitalizados; e) Demora en los servicios de radiología, laboratorio, etc.; f) Recortes del personal sanitario, particularmente de enfermería; g) Recortes del personal administrativo; $h$ ) Aumento de los problemas con el lenguaje y las barreras culturales; i) Aumento del "papeleo" burocrático-administrativo ("red tape")(Derlet, Richards, 2000; Trzeciak, Rivers, 2003; Wiess, et al, 2004; American College of Emergency Physicians, 2008; Hoot, Aronsky, 2008; Moskop, et al, 2009a; Zaragoza, et al, 2009).

Como se señaló anteriormente una de las posibles causas que explica parte de la saturación de los SUH, tiene que ver con el aumento de su utilización por parte de pacientes con PSBC, susceptibles de ser resueltos en niveles de menor complejidad del sistema asistencial. Este fenómeno viene siendo estudiado de manera significativa desde la década de los 90 del siglo pasado hasta el presente, particularmente en Estados Unidos. (Driscoll, Vincent, Wilkinson, 1987; Liggins, 1993; Afialo et al, 1995; Gill, Riley, 1996; Gill, 1999; Sanders, 2000; Sarver, et al 2002; afial, et al, 2004; Howard, et al, 2005; Northington, Brice, Zou, 2005; Carret, Fassa, Domínguez, 2009). La mayor parte de los estudios han sido realizados en Estados Unidos debido a la naturaleza de su sistema sanitario, que carece de un sistema de salud universal, por lo que la mayoría de personas de escasos recursos se ve obligada a utilizar los servicios de urgencias hospitalarios porque carecen de otra fuente de atención o de un seguro privado de salud (Rust, et, al, 2008). En Europa también se han realizado importantes estudios al respecto (Béland, et al, 1998; Murphy 1998; Pereira, et al. 2001; Bianco, Pileggi, Angelillo, 2003; Duran, et al, 2010; Gentile, et al, 2010). En el caso de España existen bastantes trabajos que dan cuenta de este fenómeno (Alonso, et al, 1993; Sempere, et al, 2001; Sánchez, Cabanillas, 2005; Sánchez, et al, 2005; Pasarín, et al, 2006, Márquez et al, 2007). Por otra parte, en España, según el Barómetro Sanitario 19952008, sólo aproximadamente un $25 \%$ de los encuestados entre el 2004 y el 2008, había acu- dido previamente al MC, es decir, el 75\% lo hizo por cuenta propia (Ministerio de Sanidad y Política Social, 2009).

Con el fin de contribuir a mejorar la comprensión sobre la utilización de los SUH por personas con PSBC, se diseñó esta investigación. Entendemos por baja complejidad aquellos problemas de salud de las personas que pueden ser resueltos, en los centros de salud de referencia, por sus respectivos MC y que son clasificados en los niveles IV y V por el Sistema de Triaje del SUH (ver Tabla 1). El objetivo central de esta investigación fue entonces comprender las razones y los motivos que impulsan a las personas con PSBC para acudir por cuenta propia y sin remisión por su $\mathrm{MC}$ al servicio de urgencias de un hospital general. Para comprender estas razones la investigación procuró determinar el peso que podían tener los factores demográficos, educativos, laborales, familiares e institucionales (expresados en términos de la relación con el centro de salud y el MC) en la decisión de acudir por cuenta propia al SUH. Además la investigación exploró la percepción sobre el grado de gravedad y preocupación atribuidos por las personas a su problema de salud. Consideramos que esta información es especialmente importante para buscar alternativas que permitan racionalizar la asistencia sanitaria tanto a nivel primario (centro de salud) como secundario y terciario (SUH) así como para evitar los problemas que genera la saturación de los SUH.

\section{METODOLOGÍA}

\subsection{DISEÑO DEL ESTUDIO}

Se realizó un estudio transversal, a través de entrevistas personales con cuestionario estructurado, mediante una muestra conveniente de pacientes, siguiendo un muestreo no probabilístico por cuotas.

\subsubsection{CONTEXTO}

La Ley General de Sanidad de 1986 definió las características principales del Sistema de Salud de España. Este sistema, de financiación pública a través de los impuestos, garantiza una cobertura universal y un acceso libre a los servi- 


\section{Tabla 1}

\section{Niveles de urgencia del triaje hospitalario}

Nivel I: Resucitación. Condición que amenaza la vida y requiere intervención inmediata

Nivel II: Emergencia. Condición que es una potencial amenaza y requiere una intervención médica rápida. Riesgo vital inmediato con inestabilidad fisiológica y/o dolor severo. Tiempo de espera máximo 15 minutos.

Nivel III: Urgencia. Condición que puede potencialmente progresar a un problema serio y que requiere múltiples exploraciones diagnósticas y/o terapéuticas en paciente con estabilidad fisiológica y/o dolor moderado. Tiempo de espera máximo 30 minutos.

Nivel IV: Semi-urgencia. Condición que implica un riesgo potencialmente serio y de complejidad significativa, constituyéndose en estándares de los servicios de urgencia hospitalarios y de alta frecuencia en atención primaria. Tiempo de espera máximo 60 minuto.

Nivel V: No urgencia. Condiciones no urgentes, problemas clínico administrativos o problemas clínicos de baja complejidad, constituyéndose en estándares de atención primaria. Tiempo de espera máximo 120 minutos.

Fuente: Modificada de: Puigblanqué, E., Noheras, Marc., Nogueras, M., Perapoch, N. (2008): El triaje en el servicio de urgencias, Enferm Clin 18(1):3-4 y Fiels, S., Lantz, F. (2006): Emergency department use by CTAS Levels IV and V patients. CJEM $8(5): 317-322$.

cios de salud. Desde 2002 se encuentra completamente descentralizado en 17 Comunidades Autónomas. Los programas, las instalaciones y los servicios de salud se organizan, geográficamente, en Áreas de Salud, con una población no menor de 200.000 ni mayor de 250.000 habitantes, y operativa y administrativamente alrededor del Equipo de Atención Primaria (EAP). El grueso de las actividades de promoción, prevención y prestación de servicios (no especializados) de la salud se realiza en los Centros de Atención Primaria o Centros de Salud, en donde los pacientes tienen asignado un(a) enfermero(a) y un (a) médico(a) de cabecera o médico(a) de atención primaria (MC). Cada Área de Salud dispondrá además de al menos un Hospital General encargado tanto del internamiento clínico como de la asistencia especializada y complementaria que requiera su zona de influencia.

El Hospital Infanta Cristina está ubicado en el Municipio de Parla que, a su vez, está localizado al sur de la Comunidad de Madrid, a unos $22 \mathrm{Km}$. de Madrid Capital. Se abrió al público a finales del mes de febrero de 2008 y es el Hos- pital General de referencia de los Municipios de Parla, Batres, Torrejón de Velasco, Torrejón de la Calzada, Cubas de la Sagra, Serranillos del Valle, Casarrubuelos y Griñón, lo que representa una población de unas 165.000 personas. En lo que lleva de funcionamiento el Hospital, es decir desde marzo de 2008 hasta diciembre de 2009, en el Hospital se han realizado 15.525 ingresos hospitalarios, 10.251 intervenciones quirúrgicas (20\% urgentes), 263.753 consultas externas especializadas, habiéndose atendido 136.293 casos de urgencia y 3.079 partos (Hospital de Parla, 2009-2010).

El Servicio de Urgencias del Hospital comenzó a funcionar a mediados del mes de abril de 2008 y se divide en urgencias generales (medicina, traumatología y cirugía), urgencias pediátricas y urgencias gineco-obstétricas. De abril a diciembre de 2008 en este servicio se atendieron 52.144 casos de urgencia (en promedio 5.794 casos mensuales y 190 casos diarios), de los cuales se ingresaron 4.102, es decir el 8\% de todos los casos atendidos. De enero a diciembre de 2009 se atendieron 84.149 casos de 
urgencias (en promedio 6.916 casos mensuales y 230 casos diarios); por otra parte, del total de personas atendidas solo el $8,4 \%$ fue ingresado y también solo el $8,5 \%$ fue remitido por el MC (Hospital de Parla, 2010).

\subsubsection{PARTICIPANTES Y CRITERIOS DE INCLUSIÓN.}

El criterio fundamental para la selección de los participantes en el estudio fue el de "problema de salud de baja complejidad". Para hacer operativo este criterio se utilizó el Sistema de Triaje del Hospital, que establece una escala de 5 niveles y unos tiempos de asistencia predefinidos, con el fin de que los pacientes sean atendidos según la gravedad que presenten a su llegada al SUH y no por el orden de llegada (Murray, Bullard, Grafstein, 2004; Fernandes, et al, 2005; Field, Lanz, 2006; Gómez, 2006; Puigblanque, Noheras, Hogueras, Perapoch, 2008) (ver Tabla 1). Se entendió entonces por pacientes con problemas de salud de baja complejidad aquellos que quedaron ubicados en los niveles IV y V de la Escala de Triaje realizado por el personal de enfermería al momento de ingresar al Servicio de Urgencias. Estos pacientes podían esperar al menos una hora antes de ser vistos por el médico de urgencias y el problema de salud por el que consultaban podía ser resuelto en su centro de salud de referencia por su médica/o de atención primaria (MAP). Los datos de 2009 mostraban que algo más del 70\% de los pacientes atendidos por el SUH se localizaron en esta franja de la Escala de Triaje. Otros criterios de selección fueron: ser mayor de 18 años, no haber sido remitido(a) por su MC, entender el español, tener las capacidades físicas y mentales para contestar adecuadamente las preguntas del entrevistador y finalmente que el motivo de consulta al servicio de urgencias no le impidiese contestar las preguntas.

\subsubsection{ESTRUCTURA DE LA ENTREVISTA}

La entrevista se realizó a través de cuestionario estructurado que constaba de 43 preguntas que incluían los siguientes aspectos de los participantes: características sociodemográficas; experiencia institucional con su servicio de salud de referencia (utilización habitual del centro de salud de referencia y grado de satisfacción y con- fianza con el médico de cabecera del centro de salud de referencia); experiencia institucional con el SUH del Hospital de Parla (utilización habitual del SUH de Hospital de Parla, problema de salud actual, utilización previa del centro de salud para resolver el problema de salud actual y razones para la utilización del SUH del Hospital de Parla para resolver el problema de salud actual), experiencia subjetiva en relación con su problema actual de salud (grado de urgencia y preocupación percibidos en relación con el problema actual de salud); valoración comparada entre el SUH del Hospital de Parla y el centro de salud de referencia (tiempo de tiempos de espera, trato de personal sanitarios, capacidad de resolución de los problemas de salud, remisión a especialistas). Para probar el cuestionario se realizó una prueba piloto antes de comenzar el estudio.

\subsubsection{RECOLECCIÓN DE LOS DATOS}

Las entrevistas se realizaron entre el 26 de abril y el 30 de julio de 2010 (14 semanas), de lunes a domingo de 10:00 a 22:00, horario de máxima afluencia de pacientes al SUH del Hospital Infanta Cristina de Parla. Teniendo en cuenta los datos históricos del SUH de 2009, se decidió seleccionar una muestra de cerca de 500 entrevistados para obtener un margen de error de \pm el $4 \%$ con un IC del $95 \%$. Para garantizar una mayor representatividad de la muestra las entrevistas se distribuyeron, siguiendo un muestreo aleatorio sistemático, teniendo en cuenta las horas (mañana, tarde, noche) y los días de la semana, a lo largo de los 3 meses que duró el estudio. Los participantes en el estudio eran captados por la enfermera del triaje de urgencias y conducidos a una sala anexa donde se le explicaba la naturaleza del estudio y se les pedía su consentimiento para realizar la entrevista a través de la firma del respectivo consentimiento informado. Las entrevistas fueron realizadas por un técnico especializado (sociólogo) debidamente entrenado, antes de que los participantes fueran vistos por el médico de urgencias.

\subsubsection{ANÁLISIS ESTADÍSTICO}

Todos los datos fueron capturados, procesados y analizados en el IBM SPSS statistic 18. Se realizó un análisis descriptivo bivariado. 


\section{RESULTADOS}

Durante el periodo del estudio se atendieron un total de 21.612 urgencias de las cuales 13.054 fueron excluidos del estudio, es decir 8.558 fueron elegibles. Al final 492 pacientes respondieron las preguntas del cuestionario, lo que significó finalmente un margen de error final de más/menos el $4,3 \%$ con un nivel de confianza del $95 \%$.

\subsection{CARACTERÍSTICAS SOCIODEMOGRÁFICAS}

Del total de entrevistados las mujeres representaron un porcentaje ligeramente superior $(53 \%)$ al de los hombres (47\%). La gran mayoría $(73 \%)$ había nacido en España y del total de extranjeros entrevistados (27\%), el 69\% nacieron en América Latina, el $22 \%$ en Marruecos y el $16 \%$ en Rumania. La media de edad de los entrevistados fue de 40 años $(\sigma=40+/-15$ años), resaltando que el $67 \%$ tenía menos de 45 años, una cuarta parte menos de 30 años y apenas el 8\% eran mayores de 65 años. Estos datos se corresponden con los datos del Padrón Municipal del Municipio de Parla, en el cual el 64\% tiene menos de 45 años y el $9 \%$ más de 65 años (Ayuntamiento de Parla, 2010). En relación con el estado civil de los entrevistados, el 52\% afirmó estar casado mientras que el 37\% manifestó estar soltero, aunque una tercera parte de los solteros afirmó encontrarse conviviendo en pareja. Sobre los vínculos familiares el 33\% manifestó no tener hijos y el $20 \%$ afirmó tener un hijo, por tanto el $47 \%$ de los entrevistados manifestó tener dos o más hijos. Resulta interesante señalar que, en términos de los vínculos familiares, sólo el 9\% manifestó vivir solo y el $67 \%$ afirmó que convivía con dos o más personas, siendo el o la cónyuge o la pareja, los hijos y los padres los que con mayor frecuencia convivían con los entrevistados. En términos educativos, el $9 \%$ no tenía estudios o tenía menos de 5 años de estudios (dos terceras partes de los cuales eran mayores de 65 años), sólo el 19\% había completado la educación primaria y tan sólo el 27\% había cursado los 10 años de educación obligatoria en España. En términos de educación para el trabajo sólo el $20 \%$ tenía for- mación profesional de Grado Medio o Grado Superior y apenas el 11\% tenía formación superior o universitaria. En términos de la actividad laboral el $56 \%$ se encontraba trabajando en el momento de ser entrevistados, de los cuales el $71 \%$ eran trabajadores manuales. De los que no trabajaban el $23 \%$ ya se había jubilado y el $43 \%$ se encontraba en el paro. Finalmente la renta media familiar mensual de los entrevistados era de 1.450 euros, resaltando que la renta familiar del $48 \%$ de los entrevistados era igual o menor a 1.500 euros. Se debe tener presente aquí que el $67 \%$ de los entrevistados viven en familias con 3 o más miembros, lo que determina que la renta per cápita familiar tienda a ser baja. De hecho, la renta media per cápita en Parla en 2007 fue de 12.157 euros año (1.013 mes), que representa el $68 \%$ de la renta media en la Comunidad de Madrid, ocupando el lugar 145 entre los 179 municipios de esta Comunidad (Instituto de Estadística, 2007). La Tabla 2 resume las principales características sociodemográficas de los participantes en el estudio.

\subsection{UTILIZACIÓN DEL CONTEXTO INSTITUCIONAL SANITARIO}

El 98\% de los entrevistados tenía tarjeta sanitaria, documento que le permite acceder de manera rutinaria y regular a los servicios de salud. Por otra parte, el $83 \%$ afirmó conocer a su médico de cabecera. En cuanto a la utilización de los servicios de salud ambulatorios (Centros de Salud), el 51\% manifestó utilizar estos servicios de manera regular y rutinaria en el último año (tres o más veces). Sólo el 18\% no lo ha utilizado el último año, fundamentalmente porque no ha tenido problemas de salud. De todas maneras, llama la atención que el $40 \%$ haya utilizado cuatro o más veces el centro de salud en el último año, teniendo en cuenta que la población es muy joven. Además, el 56\% manifestó que no utilizó este servicio en caso de urgencias y el $19 \%$ haberlo utilizado solo una vez en el último año. En otras palabras, solo el 11\% utilizó de manera más o menos regular el CS para atención de urgencias. Esto coincide con el hecho de que sólo el $34 \%$ prefiera el centro de salud en caso de urgencia o problema grave de salud. De hecho el 54\% ha utilizado los servi- 
Tabla 2

Características sociodemográficas de los participantes en el estudio

\begin{tabular}{ccc}
\hline$n=492$ & Margen de error $=+/-4,3 \%$ & Intervalo de confianza $=95 \%$ \\
\hline$n=492$ & $\%$ & $n=492 \%$
\end{tabular}

\section{Género}

Mujer

$263 \quad 53$

Hombre

$229 \quad 47$

Lugar de nacimiento

España

Otros

\section{Municipio de residencia}

Parla

Otros

\section{Grupos de edad (años)}

18-29 años

$125 \quad 25$

30-44 años

$200 \quad 41$

45-64 años

65 y más años

\section{Mayor nivel de educación}

Sin estudios

Menos de 5 años de estudio

Primaria

ESO

FP Grado Medio

FP Grado Superior

Bachillerato LOGSE

Diplomatura o Ingeniería técnica

Arquitecto/Ingeniero/Licenciado

Posgrado/Master/Doctorado

NS/NC

\section{Estado civil \\ Casada/o \\ Soltera/o \\ Viuda/o \\ Separada/o \\ Divorciada/o}

\section{Número de hijos}

$\begin{array}{lrr}\text { Ninguno } & 164 & 33 \\ 1 & 97 & 20 \\ 2 & 144 & 29 \\ 3 & 53 & 11 \\ 4 & 20 & 4 \\ \text { Más de 4 hijos } & 14 & 3\end{array}$

Número de personas con las que vive

$\begin{array}{lrr}\text { Vivo sola/o } & 43 & 9 \\ \text { Una persona } & 116 & 24 \\ \text { Dos personas } & 127 & 26 \\ \text { Tres personas } & 136 & 27 \\ \text { De cuatro a cinco personas } & 56 & 11 \\ \text { Más de cinco personas } & 14 & 3\end{array}$

\section{Situación laboral}

Trabaja

$\begin{array}{lll}\text { Trabajador manual } & 197 \quad 40\end{array}$

$\begin{array}{lll}\text { Trabajador no manual } & 77 \quad 16\end{array}$

$\begin{array}{lll}\text { Jubilado/Pensionista } & 37 & 8\end{array}$

Pensionista (no ha trabajado) $\quad 12 \quad 2$

$\begin{array}{lll}\text { Parado y ha trabajado antes } & 89 & 18\end{array}$

Parado y busca primer empleo $\quad 4 \quad 1$

Estudiante $\quad 11 \quad 2$

Trabajo doméstico no remunerado $44 \quad 9$

Otra situación $\quad 18$

$\mathrm{NS} / \mathrm{NC} \quad 3 \quad 1$

Ingresos familiares totales

Menos de $€ 300$

De $€ 301$ a $€ 600 \quad 347$

De $€ 601$ a $€ 900 \quad 52 \quad 11$

De $€ 901$ a $€ 1.200 \quad 88 \quad 18$

De $€ 1.201$ a $€ 1.500 \quad 54 \quad 11$

De $€ 1.501$ a $€ 1.800 \quad 46 \quad 9$

De $€ 1.801$ a $€ 2.100 \quad 64 \quad 13$

De $€ 2.101$ a $€ 2.400 \quad 31 \quad 6$

Más de $€ 2.400 \quad 52 \quad 10$

$\begin{array}{lll}\mathrm{NS} / \mathrm{NC} & 67 & 14\end{array}$ 
cios de urgencias de Hospital de Parla dos o más veces en el último año. Llama también la atención que un $21 \%$ haya utilizado este servicio de urgencias más de tres veces en el último año. Finalmente es interesante resaltar que el $60 \%$ de los entrevistados afirmó que normalmente le dan hora con su médico de cabecera el mismo día que la solicita $(18 \%)$ o al día siguiente (42\%). La Tabla 3 resume las principales características de la utilización del contexto institucional sanitario por los entrevistados.

\subsection{PERCEPCIÓN EN RELACIÓN CON EL PROBLEMA ACTUAL DE SALUD POR EL QUE SE ACUDE AL SUH}

Para el 50\% de los entrevistados el problema por el que acude a urgencias fue considerado poco grave $(42 \%)$ o nada grave $(9 \%)$. En este mismo sentido el $70 \%$, consideró que su situación no es grave pero sí requiere atención urgente o rápida, hecho que también se ve reflejado por el tiempo que creen que deben esperar, de tal manera que el $53 \%$ pensó que debe ser atendido en menos de 30 minutos y el $33 \%$ en menos de 1 hora. Finalmente, para el 65\% de los entrevistados el problema de salud por el que acudió a urgencias les generó bastante preocupación, puesto que en una escala de 0 a 10 , donde 0 es nada de preocupación y 10 mucha preocupación calificaron esta preocupación con 7 o más. La media de preocupación fue de 6,71. La Tabla 4 resume la percepción de los entrevistados en relación con el problema actual de salud por el que se acude al SUH.

\subsection{RAZONES PARA UTILIZAR EL SUH POR CUENTA PROPIA}

El 63\% de los entrevistados consideró que su problema de salud no se lo hubiese podido resolver su médico de cabecera, en el supuesto de que los atendiera en ese mismo instante. Por otra parte el $73 \%$ no buscó alternativa de atención médica, previamente a su desplazamiento al servicio de urgencias, es decir, no consultó a su médico de cabecera. A los entrevistados que decidieron acudir por cuenta propia al SUH se le dio a escoger, entre varias opciones, la razón o razones (podían escoger una o más opciones) para haber tomado esta decisión. En este sentido del $73 \%$ que no acudió previamente al médico de cabecera, un $42 \%$ consideró que este servicio cuenta con mejores medios técnicos para resolver el problema de salud que el CS; además un $22 \%$ también se desplazó por sus propios medios porque consideró que en el SUH resuelven con más rapidez su problema de salud. Para el 55\% de los entrevistados la comodidad, en términos de horario o de vivir cerca del hospital, fue también una de las razones fundamentales que los impulsó a acudir al SUH por cuenta propia. A los que acudieron previamente al médico de cabecera (27\%), también se les dio a escoger entre varias opciones la razón o razones (podían escoger una o más opciones) para haber tomado la decisión de acudir después al SUH. La falta de eficacia del tratamiento $(71 \%)$, la necesidad de tener otra opinión $(39 \%)$ y la sugerencia por parte de su médico de cabecera de acudir a urgencias en caso de no mejoría (26\%) fueron las principales razones que los impulsó a acudir por sus propios medios al SUH. La Tabla 5 resume las razones de lo entrevistados para utilizar el SUH por cuenta propia.

\subsection{VALORACIÓN COMPARATIVA ENTRE EL SUH Y EL CS}

El $56 \%$ de los entrevistados manifestó tener un alto grado de satisfacción con la atención que le brinda su médico de cabecera, en la medida en que calificó con 8 o más su grado de satisfacción en una escala de 0 a 10 , donde 0 es nada satisfecho y 10 muy satisfecho. Sólo el $8 \%$ manifestó niveles bajos de satisfacción (insatisfacción) al calificar con menos de 5 en la misma escala. De cualquier manera la media de satisfacción fue alta $(7,78+/-2,49)$. En términos del grado de confianza que le tienen a la atención que les brinda el médico de cabecera, el 59\% de los entrevistados manifestó tener un alto grado de confianza con esta atención, en la medida en que calificó con 7 o más su grado de confianza en una escala de 0 a 10 , donde 0 es nada de confianza y 10 mucha confianza. De igual manera que el grado de satisfacción con la atención, sólo el 13\% manifestó niveles bajos de confian- 
Tabla 3

Características de la utilización del contexto institucional sanitario

\begin{tabular}{lcr}
\hline \multicolumn{1}{c}{ Margen de error $=+/-\mathbf{4 9 \%}$} & Intervalo de confianza $=\mathbf{9 5 \%}$ \\
\hline & $\mathbf{n = 4 9 2}$ & $\%$ \\
\hline ¿Tienen tarjeta sanitaria? & & \\
SI & 480 & 98 \\
NO & 12 & \\
¿Conoce a su médico de cabecera & & 83 \\
SI & 409 & 17 \\
No & 83 &
\end{tabular}

En el último año, ¿cuántas veces ha acudido a su ambulatorio o centro de salud por algún problema de salud no urgente?

$\begin{array}{lrr}\text { Ninguna } & 89 & 18 \\ \text { Una vez } & 59 & 12 \\ \text { Dos veces } & 89 & 18 \\ \text { Tres veces } & 54 & 11 \\ \text { Cuatro o más veces } & 197 & 40 \\ \text { NS/NC } & 5 & 1\end{array}$

En el último año, ¿cuántas veces ha acudido a su ambulatorio o centro de salud por algún problema grave o urgente?

$\begin{array}{lrr}\text { Ninguna } & 276 & 56 \\ \text { Una vez } & 93 & 19 \\ \text { Dos veces } & 64 & 13 \\ \text { Tres veces } & 20 & 4 \\ \text { Cuatro o más veces } & 34 & 7 \\ \text { NS/NC } & 5 & 1\end{array}$

En caso de padecer algún problema de salud que considere urgente, ¿a dónde preferiría ir en primer lugar?

A las urgencias de mi centro de salud para tener una primera opinión $\quad 156 \quad 32$

A las urgencias del Hospital de Parla directamente $\quad 325 \quad 66$

$\mathrm{NS} / \mathrm{NC}$

En el último año, ¿cuántas veces ha acudido a las urgencias del hospital de Parla?
Es la primera vez
Dos veces
Tres veces
$\mathrm{NS} / \mathrm{NC}$

¿Cuánto tiempo tardan habitualmente en darle hora con su médico de cabecera?

Normalmente el mismo día

$\begin{array}{rr}88 & 18 \\ 206 & 42 \\ 57 & 12 \\ 24 & 5 \\ 21 & 4 \\ 96 & 19 \\ 96 & 19\end{array}$


za (desconfianza) al calificar con menos de 5 en la misma escala. También en este caso la media de confianza fue alta $(7.38+/-2.81)$. Cuando se le pidió a los entrevistados que evaluaran comparativamente el SUH con su CS en varios aspectos (tiempo de espera en ser atendido, trato del personal médico y de enfermería, confianza que le produce el personal sanitario, información sobre el problema de salud, capacidad para resolver los problemas de salud y tiempo de derivación al especialista) se encontró que entre el $33 \%$ y el $37 \%$ tenía dificultades para realizar tal evaluación, sea porque no conocen a su médico de cabecera $(17 \%)$ o porque es la primera vez que acudían al SUH en el último año (46\%). Hay que recordar que el SU del Hospital de Parla comenzó a funcionar apenas en abril de 2008. Con esta aclaración sólo el $25 \%$ de los

Tabla 4

Percepción en relación con el problema actual de salud por el que se acude al servicio de urgencias hospitalaria

\begin{tabular}{lcr}
\hline $\mathbf{n}=\mathbf{4 9 2}$ & Margen de error $=+/-\mathbf{4 , 3} \%$ & Intervalo de confianza $=\mathbf{9 5 \%}$ \\
\hline & $\mathbf{n}=\mathbf{4 9 2}$ & $\%$ \\
\hline Valore el nivel de gravedad por el que acude al Hospital de Parla & & \\
Muy grave & 23 & 5 \\
Grave & 202 & 41 \\
Poco grave & 201 & 41 \\
Nada grave & 45 & 9 \\
NS/NC & 21 & 4
\end{tabular}

¿Cuál de las siguientes frases expresan mejor la sensación que tienen en relación con el problema de salud por el que acude al Hospital de Parla?

Creo que si no me atienden inmediatamente mi vida está en peligro

Creo que si no me atienden inmediatamente el problema puede ir a más

$\begin{array}{rr}9 & 2 \\ 130 & 26 \\ 220 & 45 \\ 102 & 21\end{array}$

No creo que sea grave pero quiero que me lo resuelvan rápido

$\mathrm{NS} / \mathrm{NC}$ mis problemas de salud

¿Cuánto tiempo cree que debe esperar para recibir atención médica por el problema de salud por el que acude al Servicio de Urgencias del Hospital de Parla?

Inmediatamente

$\begin{array}{rr}60 & 12 \\ 201 & 41 \\ 163 & 33 \\ 38 & 8 \\ 14 & 3 \\ 16 & 3\end{array}$

De 15 a 30 minutos

Hasta una hora

Hasta dos horas

Más de dos horas

Califique de 0 a 10 el grado de preocupación por el problema de salud por el que acude al Servicio de Urgencias del Hospital de Parla?

\begin{tabular}{cccccccccccccc} 
Nada preocupado & $\mathbf{0}$ & $\mathbf{1}$ & $\mathbf{2}$ & $\mathbf{3}$ & $\mathbf{4}$ & $\mathbf{5}$ & $\mathbf{6}$ & $\mathbf{7}$ & $\mathbf{8}$ & $\mathbf{9}$ & $\mathbf{1 0}$ & NS/NC & Muy preocupado \\
$\mathbf{n}=\mathbf{4 9 2}$ & 21 & 6 & 12 & 17 & 21 & 54 & 47 & 96 & 109 & 41 & 65 & 3 & $\mathbf{n = 4 9 2}$ \\
$\mathbf{\%}$ & 4 & 1 & 2 & 4 & 4 & 11 & 10 & 20 & 22 & 8 & 13 & 1 & $\%$ \\
\hline & 6 & & & & & & & & & & & & $\%$
\end{tabular}

Media: 6,71 $\sigma=2,51$ 
Tabla 5

Razones para la utilización del servicio de urgencias hospitalaria por cuenta propia

\begin{tabular}{ccc}
\hline$n=492$ & Margen de error $=+/-4,3 \%$ & Intervalo de confianza $=95 \%$ \\
\hline & $n=492$ & $\%$ \\
\hline
\end{tabular}

¿El problema de salud se lo podrían haber resuelto en su Centro de Salud en caso de ser atendido en este momento?

SI

$120 \quad 24$

NO

$309 \quad 63$

$\mathrm{NS} / \mathrm{NC}$

$63 \quad 13$

$\mathrm{n}=133 \quad \% *$

¿Acudió a su ambulatorio o Centro de Salud por este problema d salud antes de acudir a las urgencias del hospital?

SI $133 \quad 27$

$\mathrm{NO}$

Si ya acudió a su Centro de Salud por este problema, ¿por qué acude a las urgencias del Hospital de Parla?*

Porque el tratamiento que me dio/recetó no me mejoró y decidí venirme a urgencias.

$94 \quad 71(44)$

Porque no estoy satisfecho con el tratamiento que me recetaron/dieron en el Centro de Salud y quiero una segunda opinión.

Porque me dijo que si no mejoraba con el tratamiento que me dio/recetó viniera directamente a urgencias.

Porque en el Centro de Salud no me remitieron al especialista y espero que en urgencias me vea el especialista.

Porque en el ambulatorio no me pidieron pruebas (analítica, RX. TAC, Resonancia, etc.). $17 \quad 13$ (7)

\section{¿Por qué no acudió a su Centro de Salud antes de acudir a las urgencias del Hospital de Parla?*}

Porque en la urgencia cuentan con más y mejores medios técnicos para resolver los problemas de salud que en el centro de salud.

Porque en la urgencia resuelven más rápidamente los problemas de salud que en el centro de salud.

Porque me es más cómodo venir al hospital que al centro de salud (cercanía, horario, etc.). $63 \quad 20$ (12)

$\begin{array}{llll}\text { Porque creía que me iba a mejorar y como empeoré me vine directamente a urgencias. } & 31 & 10(6)\end{array}$

Porque mi médico de cabecera me dijo que cuando tuviera este problema me fuera directamente a urgencias.

Porque ya había venido a urgencia y me dijeron que volviera si no mejoraba.

Porque tardan mucho tiempo en darme hora con mi médico de cabecera y por eso me vine a urgencias

Porque tuve un accidente (de trabajo, doméstico, etc.) 12

Porque llevo mucho tiempo con este problema y no me lo resuelve mi médico de cabecera. $\quad 9 \quad 3$ (2)

Otros

\footnotetext{
* Pregunta de elección múltiple. Las cifras sin paréntesis hacen referencia al porcentaje de entrevistados que escogieron esta alternativa (porcentaje absoluto). Las cifras entre paréntesis corresponden a porcentajes relativos en relación con el total de respuestas.
} 
Tabla 6

Percepción en relación con el problema actual de salud por el que se acude al servicio de urgencias hospitalaria

\begin{tabular}{lll}
\hline$n=492$ & Margen de error $=+/-4,3 \%$ & Intervalo de confianza $=95 \%$ \\
\hline
\end{tabular}

¿Califique de 0 a 10 el nivel de satisfacción con la atención que le brinda su médico de cabecera?

$\begin{array}{cccccccccccccc}\text { Nada satisfecho } & \mathbf{0} & \mathbf{1} & \mathbf{2} & \mathbf{3} & \mathbf{4} & \mathbf{5} & \mathbf{6} & \mathbf{7} & \mathbf{8} & \mathbf{9} & \mathbf{1 0} & \mathbf{N S} / \mathbf{N C} & \text { Muy satisfecho } \\ \mathbf{n = 4 9 2} & 12 & 4 & 7 & 9 & 10 & 25 & 15 & 48 & 77 & 72 & 122 & 91 & \mathbf{n = 4 9 2} \\ \mathbf{\%} & 2 & 1 & 1 & 2 & 2 & 5 & 3 & 10 & 16 & 15 & 25 & 18 & \mathbf{\%}\end{array}$

Media: $7,78 \sigma=\mathbf{2 , 4 9}$

¿Califique de 0 a 10 el nivel de confianza con la atención que le brinda su médico de cabecera?

$\begin{array}{cccccccccccccc}\begin{array}{c}\text { Nada de confianza } \\ \mathbf{n}=\mathbf{4 9 2}\end{array} & \mathbf{1} & \mathbf{2} & \mathbf{3} & \mathbf{4} & \mathbf{5} & \mathbf{6} & \mathbf{7} & \mathbf{8} & \mathbf{9} & \mathbf{1 0} & \mathbf{N S} / \mathbf{N C} & \begin{array}{c}\text { Mucha confianza } \\ \mathbf{0}=\mathbf{4 9 2}\end{array} \\ \mathbf{\%} & 4 & 1 & 1 & 2 & 3 & 7 & 4 & 10 & 14 & 10 & 25 & 19 & \mathbf{\%}\end{array}$

Media: $7,38 \sigma=\mathbf{2 , 8 1}$

Comparación del Servicio de Urgencias (SU) del Hospital de Parla con su Centro de Salud (CS)

Tiempo que tiene que esperar para ser atendido Mejor en el SU del H de Parla que en el CS Igual en el SU del H de Parla que en el CS Peor en el SU del $\mathrm{H}$ de Parla que en el CS $\mathrm{NS} / \mathrm{NC}$ $\mathrm{n}=492 \quad \mathbf{7 \%}$

Trato del persona médico

Mejor en el SU del H de Parla que en el CS Igual en el SU del H de Parla que en el CS Peor en el SU del $\mathrm{H}$ de Parla que en el CS $\mathrm{NS} / \mathrm{NC}$

Trato del personal de enfermería Mejor en el SU del $\mathrm{H}$ de Parla que en el CS Igual en el SU del $\mathrm{H}$ de Parla que en el CS Peor en el SU del $\mathrm{H}$ de Parla que en el CS $\mathrm{NS} / \mathrm{NC}$

$\begin{array}{rr}74 & 15 \\ 51 & 10 \\ 207 & 42 \\ 160 & 33 \\ & \\ 84 & 17 \\ 188 & 38 \\ 58 & 12 \\ 162 & 33 \\ & \\ 84 & 16 \\ 195 & 40 \\ 33 & 7 \\ 185 & 37 \\ & \\ 112 & 23 \\ 139 & 28 \\ 75 & 15 \\ 166 & 34 \\ & \\ 120 & 25 \\ 133 & 27 \\ 61 & 12 \\ 178 & 36 \\ & \\ 248 & 50 \\ 59 & 12 \\ 17 & 4 \\ 168 & 34 \\ & \\ 134 & 27 \\ 99 & 20 \\ 18 & 49 \\ 241 & 49\end{array}$


entrevistados consideró que en el tiempo de espera en el SU era igual (15\%) o mejor $(10 \%)$ en relación con su CS; el 55\% consideró que el trato del personal médico del SU era mejor $(17 \%)$ o igual (38\%) en relación con su CS; el $56 \%$ consideró que el trato del personal del enfermería del SU era mejor (16\%) o igual (12\%) en relación con su CS; el 51\% consideró que la confianza que le genera el personal sanitario del SU era mejor (23\%) o igual $(28 \%)$ en relación con su $\mathrm{CS}$; el $52 \%$ consideró que la información que le suministraban sobre su problema de salud en el SU era mejor (25\%) o igual $(27 \%)$ en relación con su CS; el $62 \%$ consideró que la capacidad para resolver los problemas de salud del SU era mejor (50\%) o igual $(12 \%)$ en relación con su CS; finalmente el $47 \%$, consideró que el tiempo de derivación al médico especialista del SU era mejor (27\%) o igual $(20 \%)$ en relación con su CS. En esta última pregunta el $49 \%$ de los entrevistados tuvo dificultad en realizar esta evaluación. La Tabla 6 resume la valoración comparativa entre el SU el CS

\section{DISCUSIÓN}

El objetivo principal de este estudio era conocer y comprender las razones y los motivos que dan las personas con problemas de baja complejidad para acudir por cuenta propia al SU de un Hospital General. En este sentido, el estudio tuvo en cuenta la perspectiva del paciente a la hora de definir como urgente su problema de salud (criterios subjetivos). Sin embargo utilizó criterios explícitos de inclusión en el estudio, a través del sistema de triaje hospitalario, para definir la complejidad del problema de salud. Para tratar de comprender estas razones, el estudio exploró las características sociodemográficas, la experiencia institucional sanitaria previa, la percepción de la gravedad y la preocupación que generaba el problema de salud y, finalmente, una valoración comparativa entre el SUH y el CS.

Como se señaló al comienzo del artículo, España cuenta con un sistema nacional de salud que garantiza el acceso gratuito y universal de todos los ciudadanos a la asistencia sanitaria, tanto a nivel primario (CS) como a nivel secundario y especializado (Hospitales). Esta garantía se institucionaliza a través de la denominada tarjeta sanitaria, que, como puso en evidencia el estudio, la poseía el $98 \%$ de los entrevistados, independientemente de que el $27 \%$ tuviese una nacionalidad diferente a la española. De todas maneras es importante remarcar que el hecho de no poseer tarjeta sanitaria no impide el uso libre y gratuito de los servicios de urgencia tanto del CS como de los SUH. Este es un hecho central para la interpretación de los resultados en el caso de España porque, como señalan algunos autores, la falta de un servicio de atención en salud habitual se convierte en un factor importante que predispone al uso de los SUH por problemas de salud de baja complejidad (Afilalo, et al, 1995; Rubin, Bonnin, 1995; Gill, et al, 2000; Sarver, et al, 2002; Sánchez, Cavanillas, 2005; Han, et al, 2007; Lowthian, et al, 2010; Tranquada, et al, 2010).

Casi en la misma proporción de lo que ocurre en el resto de España, el $73 \%$ de los entrevistados no acudió previamente a su MC. Dos fueron las principales razones que adujeron para acudir directamente y por cuenta propia al SUH. En primer lugar la mejor eficacia y efectividad, en términos de recursos técnicos y rapidez, para resolver el problema de salud y, en segundo lugar, la comodidad, en términos de horarios y accesibilidad. El 27\% restante, que acudió en primera instancia a su MC, decidió acudir posteriormente a urgencias fundamentalmente porque el tratamiento no fue efectivo y quería una segunda opinión. En este caso parece que la eficacia y la efectividad que proyecta el SUH también están en la base de las razones que dieron los que acudieron previamente a su MC. Es interesante constatar además que, aunque en un primer momento, el $50 \%$ de los entrevistados manifestó que el problema de salud por el que había acudido al SU era poco o nada grave, finalmente reconoció que consideraba que su problema no era grave, pero lo que sí quería era una solución urgente o rápida a dicho problema. Esto se correlaciona bastante bien con el hecho de que el $53 \%$ espera ser atendido en menos de media hora y el 33\% en menos de 1 hora, independientemente del tiempo que les asignó el triaje hospitalario. Si consideramos además que para el $63 \%$ el problema de salud les genera bastante preocupación -es decir, aquello que calificaron con 7 o más de 7 su grado de preocupación en una escala de 0 a 10 - todo parece indicar 
que es la necesidad de resolver rápida, efectiva y eficientemente un determinado problema de salud, más que su sensación de gravedad, la que está en la base de las razones que movilizan a los paciente a acudir a urgencias por cuenta propia al SUH.

De acuerdo con los resultados esta forma de razonar puede tener que ver, en buena parte, con la experiencia institucional del paciente con su CS. Efectivamente, estamos hablando de entrevistados que, en términos generales, a) son usuarios regulares de su CS, puesto que el 51\% ha utilizado tres o más veces el CS en el último año, b) que mayoritariamente (66\%) cree que su MC no les hubiese podido resolver el problema de salud en caso de que los hubiese atendido en ese momento y c) que también consideran que el SUH tiene mayor capacidad para resolver los problemas de salud, en relación con el CS $(50 \%$ del total o $77 \%$ si se considera solamente aquellos que contestaron esta pregunta, es decir, $66 \%$ de los entrevistados). Además, parece bastante claro que la idea de respuesta rápida se relaciona más con el tiempo que dura la resolución total del problema de salud que con los tiempos de espera en ser atendidos. En efecto, el $60 \%$ de los entrevistados reconoce que habitualmente le dan hora con su MC el mismo día (18\%) o al día siguiente $(40 \%)$ de solicitarla. Esta impresión parece reforzarse con el hecho de que el punto donde peor sale evaluado el SUH en relación con el CS es el relacionado con el tiempo en ser atendido.

En relación con la experiencia institucional, los datos parecen poner en evidencia que existen altos niveles de satisfacción y confianza con la atención que brinda, en términos generales, el MC del respectivo CS y que no parecen existir grandes diferencias cuando se compara tanto el trato que brinda como la confianza y la información que da el personal sanitario, tanto en el CS como en el SUH. Sin embargo, como se señaló anteriormente, sí existe una diferencia muy significativa cuando se evalúa la capacidad para resolver el problema de salud por el que se consulta. Esto parece indicar que la experiencia que tienen los pacientes en su CS les ha permitido desarrollar una visión bastante selectiva en cuanto a la resolución de los problemas de salud. Esto quiere decir que para los pacientes el CS está para resolver un tipo particular de problemas que, en términos generales, evalúan satisfactoriamente, pero que para resolver problemas de salud que les preocupa hay que acudir a los SUH. Se podría plantear la hipótesis de que, desde la perspectiva del paciente, el CS resuelve satisfactoriamente bien ciertos problemas de orden administrativo burocrático (recetas, bajas y altas médicas, informes médicos, etc.) o de control y seguimiento (pruebas analíticas, toma de tensión arterial, seguimiento de INR, control de la glucemia, curaciones, seguimiento de tratamientos, etc.). Ahora bien, si el problema de salud genera cierta preocupación o es de cierta complejidad, también desde la perspectiva del paciente, es preferible acudir al SUH en términos de eficacia, efectividad y rapidez de resolución. Aquí parece jugar un papel importante el tiempo de resolución del problema puesto que el paciente, al percibir al CS como una institución con cierto funcionamiento administrativo burocrático, también percibe que la resolución de su problema de salud seguirá este funcionamiento, mediado por los tiempos de espera que implica realizar un determinado procedimiento: tiempo que hay que esperar para realizarlo, para pedir y para que le den nueva hora con el MC para conocer los resultados, para que le den hora en caso dado con el especialista o para un nuevo procedimiento, etc. Es por esto que el hecho de contar con una fuente de atención médica diferente al $\mathrm{SUH}$, como es el caso de España, no reduce de suyo la utilización inadecuada de los SUH como lo señalan varios autores (Burnett, Grover, 1996; Gill, Riley, 1996; Afialo et al, 1995; Boushy, Dubinsky, 1999; Oterino de la Fuente, et al, 2006; Field, Lantz, 2006; Backman, 2008; Rust, et al, 2008)

Tanto los resultados de este estudio como su interpretación coinciden con los encontrados por otros autores en el caso de España. Para Alonso, et al (1993) es un hallazgo generalizado el uso excesivo de los SUH por problemas de salud que no justifican tal uso. Para estos autores parte de este excesivo uso depende de la burocratización y masificación de la atención primaria, la falta de información sobre los recursos sanitarios, la mayor confianza de los usuarios por las tecnologías, la gran accesibilidad a los SUH por la ausencia de trámites burocráticos, por la comodidad de horarios, etc. Vázquez, et al (2000) en un estudio basado en entrevistas 
telefónicas realizadas a pacientes que acudieron por cuenta propia al SUH del Hospital 12 de Octubre de Madrid encontraron que el 32\% lo hizo por desconocimiento de urgencias extrahospitalarias, el $27 \%$ por el empleo de mejores medios técnicos, el $21 \%$ por la asistencia más rápida y el $11 \%$ por la sensación de urgencia vital. Aranaz, et al (2006) en un estudio realizado a través de encuestas a pacientes que utilizaron por cuenta propia el SUH del Hospital Universitario de Alicante encontraron que el 35\% lo hizo porque lo atienden más rápidamente o les resulta más cómodo, el 30\% lo hizo porque le da más confianza el hospital y el $22 \%$ porque cree precisar pruebas diagnósticas y piensan que en el hospital se las harán. Finalmente en un interesantes estudio cualitativo realizado en Cataluña a través de grupos focales de discusión, Pasarín et al (2006) concluyen que las personas valoran especialmente los SUH por la tecnología y el servicio profesional del personal sanitario, la exhaustividad tanto en la historia clínica como en la exploración física, por las pruebas complementarias y por la inmediatez de la resolución del problema. En este mismo estudio las personas valoraron negativamente los largos tiempos de espera pero que finalmente los justificaban si se los comparaba con los tiempos de resolución del proceso si se hubiese escogido un servicio no hospitalario (tiempo entre derivaciones, pruebas complementarias, etc.).

De igual manera los resultados de este estudio coinciden con estudios realizados en otros países. Es así como Gentile, et al (2010) siguiendo la misma metodología de este estudio (mayores de 18, que acudieron por cuenta propia al servicio de urgencias y ser clasificados como no urgentes por el triaje hospitalario) encontraron una media de edad de $36+/-12$ años, que el $71 \%$ tenía médicos de cabecera, el $76 \%$ acudió directamente al hospital, el $66 \%$ lo hizo por dolor, el $38 \%$ lo hizo por recursos diagnósticos y el $22 \%$ porque no pudieron acceder al MC. Field y Lantz (2006), utilizando una metodología semejante encontraron las siguientes razones dadas por los pacientes para haber acudido por cuenta propia al SUH: $49 \%$ porque necesitaban un servicio específico (en la mayoría de los casos, rayos $\mathrm{X}$ ), el 43\% necesitaba tratamiento urgente (rápido) y el $23 \%$ no podía utilizar los servicios del médico de cabecera en ese momento. De igual manera Northington et al (2005) siguiendo una metodología semejante encontraron que el $75 \%$ de los pacientes acudieron directamente por cuenta propia al SUH y las razones que dieron los que participaron en el estudio fueron las siguientes: $76 \%$ mejor atención médica, $74 \%$ atención urgente, $69 \%$ atención inmediata, y $40 \%$ comodidad. Cuando compararon el SUH con su fuente habitual de atención consideraron que el SUH era mejor por su flexibilidad de horario. Es importante tener en cuenta que en este estudio solo el $44 \%$ tenía médicos de cabecera. Afilalo et al (2004), encontraron que, entre los pacientes clasificados en 5 en la escala de triaje, que tenían MC y que acudieron directamente al SUH lo hicieron porque en ese momento el MC no los podía atender por razones de horario (32\%), porque consideraban que su problema era grave $(22 \%)$ y por confianza o familiaridad con el SUH (19\%). Por su parte Han, et al (2007) encontraron que el $89 \%$ de aquellos pacientes que tenían problemas de salud de baja complejidad y que a la vez tenían al menos una alternativa de atención consideraron al SUH como la mejor opción.

Desde el punto de vista de la influencia de los factores sociodemográficos en el comportamiento de las personas con problemas de salud de baja complejidad que utilizan los SUH por cuenta propia, los datos permiten tipificar al tipo de usuario. Hablamos en primer lugar de personas jóvenes con una media de edad de 40 años $(\sigma=40+/-15$ años), de las cuales el $25 \%$ tenía menos de 30 años y sólo el $8 \%$ tenía 65 años o más. Este hecho parece corroborar los resultados de varios trabajos recientes que han puesto en evidencia que el uso inapropiado de las urgencias es más frecuente entre personas jóvenes (Lang, et al, 1997; Lee, et al, 2000; Pereira, et al. 2001; Sempere-Selva, et al, 2001; Sarver, Cydulka, Baker, 2002; Bianco, Pileggi, Angeli1lo, 2003; Oktay, et al, 2003; Afiealo, et al, 2004; Aranaz, et al 2006; Carret, Fassa, Kawachi, 2007; Carret, Fassa, Domingues, 2009). Desde una perspectiva epidemiológica, a pesar de que esta distribución de las personas que participaron en el estudio refleja la distribución de la población de Parla, cabría esperar de todas maneras que acudieran al SUH más personas mayores de 65 años. Como lo reflejan las cifras internacionales del 12 al $24 \%$ de las personas 
que acude al SUH son mayores de 65 años (Strange, Chen, 1998; Salvi, et al, 2007; Lowthian, 2010; Samaras, et al, 2010).

Por otra parte, en la medida en que apenas el $9 \%$ de los entrevistados manifestó vivir solo, no parece que este hecho sea un factor que movilice activamente al uso del SUH. Como señala Carret, et al (2009) en una revisión exhaustiva, la mayoría de los estudios no parecen encontrar una asociación clara entre nivel de estudios, el estatus ocupacional y el nivel de ingresos con el uso inadecuado del SUH. En nuestro caso el estatus ocupacional y el nivel de ingresos se corresponde con un estrato socioeconómico medio-bajo en la medida en que la mayoría de las personas que trabajan realizaban trabajos manuales y la media de ingresos familiares era de 1.450 euros mensuales; sin embargo creemos que es necesario realizar estudios comparativos para poder evaluar más cuidadosamente el peso que tienen los factores socioeconómicos a la hora de utilizar los SUH por problemas de salud de baja complejidad, pues nuestro estudio ha puesto más el énfasis en la experiencia institucional previa que en estas condiciones

Finalmente, la limitación principal de este estudio es que incluye solo un hospital general, muy circunscrito espacial (incluye una zona muy específica del sur de la comunidad de Madrid) y temporalmente (el SU lleva menos de 3 años de funcionalmente) por lo que puede resultar muy difícil extrapolar los resultados al resto de España. No obstante, estudios previos realizados en España y en otros países indican que los resultados parecen recoger en términos generales un comportamiento más o menos típico, en relación con personas que acuden por cuenta propia al SUH por problemas de salud de baja complejidad. Por otra parte es necesario realizar estudios comparativos para poder evaluar con más precisión los factores que influyen en ese comportamiento, particularmente los sociodemográficos y los institucionales. También parte este estudio de recoger la opinión de pacientes clasificados en la escala de 4 y 5 del triaje hospitalario, previamente a la prestación de la atención médica. En este sentido nuestro estudio no permitió conocer qué porcentaje de esos pacientes tenía realmente un problema de salud de baja complejidad, desde la perspectiva del personal sanita- rio, una vez fueron atendidos. Este dato resulta muy importante para saber con más precisión si el triaje hospitalario ( 4 y 5) es sensible para seleccionar pacientes con problemas de salud de baja complejidad. Finalmente, al ser un SUH que entró en funcionamiento hace relativamente poco tiempo resultó muy difícil recoger la valoración comparativa de los entrevistados en relación con su CS. Por esto un porcentaje importante de los entrevistados (la tercera parte o más) no fue capaz de realizar esta evaluación.

\section{CONCLUSIONES}

La mejor eficacia y efectividad para resolver los problemas de salud, en términos de gestión de recursos técnicos y del tiempo, y la comodi$\mathrm{dad}$, en términos de horarios, disponibilidad y accesibilidad, parecen ser las principales razones por las cuales las personas con problemas de salud de baja complejidad utilizan por cuenta propia los SUH. La preocupación, más que la gravedad, parece movilizar activamente la búsqueda de solución al problema de salud de manera rápida y urgente. De allí la importancia de la eficacia, de la efectividad y de la comodidad. Estas razones parecen sustentarse, al menos en una parte significativa, en la experiencia institucional previa con el CS el cual genera alta confianza y satisfacción, en términos generales, para ciertos problemas de salud que no generen especial preocupación y que no requieran acciones en el corto plazo para ser solucionadas.

Es importante resaltar que los resultados de la investigación coinciden con los hallazgos de otras investigaciones realizados tanto en España como en otros países de Europa y de Norte América (Estados Unidos y Canadá).

A partir de los resultados de la investigación se pueden proponer algunas alternativas que podrían contribuir a reducir la utilización del servicio de urgencias por personas con problemas de salud de baja complejidad. Estas alternativas pasan, en lo fundamental, por la canalización de la trayectoria terapéutica de los pacientes, a partir de una clara definición de las expectativas tanto en relación con la atención como con los resultados esperados en el SUH. 
Para esto es necesario realizar una clara formalización de la estructura y funciones del Servicio de Urgencias, de cara al usuario, de tal forma que, desde el ingreso al Servicio, tenga claras las posibles trayectorias terapéuticas dentro del SUH y los resultados que puede esperar. Esto supone concretamente el desarrollo de una cultura del uso del SUH. Abordar la utilización del SUH a partir del desarrollo de una cultura del uso, se basa en dos hechos fundamentales encontrados en el estudio: 1) que la mayoría de los pacientes reconocen que su demanda no es urgente y que es más la preocupación la que está en la base de esta demanda; 2) que sus expectativas con respecto a la institución, están mediadas por sus percepciones subjetivas en relación con el funcionamiento del servicio, comparativamente hablando con su CS, en términos de eficacia y efectividad, y la comodidad atribuida, en términos de cercanía geográfica y de horarios de atención.

En un escenario institucional ideal, las personas que acudan al SUH deberían tener claro, desde el ingreso al servicio, los siguientes aspectos:

1) Que su demanda será atendida, siempre y cuando sea un problema de salud que requiera atención, así sea un problema de salud de baja complejidad. Esto podría significar que algunas demandas no fuesen atendidas porque resultasen francamente inadecuadas. Algunas de estas demandas podrían ser: solicitud de vacunación rutinaria, solicitud de pruebas de embarazo o de confirmación de embarazo, solicitud de recetas. De cualquier manera, se deberían formalizar estos casos, e incluso llegar a un consenso con los CS.

2) Que la demanda será canalizada a través de un sistema de clasificación conocido como triaje hospitalario, que define la gravedad de la demanda en función de unos tiempos de espera promedios antes de recibir la atención. Esto significaría que las personas tuviesen claro que la atención no es una función del orden de llegada sino de la gravedad expresada en tiempos de espera. En este sentido se debería tener claro que personas que han acudido posteriormente al SUH puedan ser atendidas antes, priorizándose siempre los paciente críticamente enfermos y los pacientes que acuden a través de ambulancia.
3) Que la atención de su demanda, en términos tanto de la trayectoria terapéutica en el SUH como del resultado de esa atención, no depende exclusivamente de la experiencia subjetiva de la persona demandante y que inclusive puede ser independiente de esa experiencia subjetiva. La persona debería tener claro que su demanda, en términos de experiencia subjetiva, es canaliza$\mathrm{da}$, en términos médicos, por el facultativo que la atiende, teniendo como referencia criterios de urgencia médica. En este sentido la atención de la demanda significa, en primer lugar, su tramitación en términos de gravedad y, en segundo lugar, de diagnóstico. Esto significa que, una vez descartadas con alta probabilidad la urgencia y gravedad de la demanda, tanto el diagnóstico como el tratamiento último se puede establecer en el propio SUH, postergarse a una atención ambulatoria especializada o al seguimiento por parte del MAP.

4) Que los tiempos de atención a la demanda dependen, no solamente de la experiencia subjetiva de la persona demandante, sino de su tramitación, en términos médicos, por parte del facultativo tratante, de la mayor o menor gravedad de la demanda de otras personas que están siendo tratadas simultáneamente y de la saturación del servicio.

Evidentemente, resulta difícil aproximarse a este escenario ideal institucional. Sin embargo, su explicitación y formalización en términos de prácticas y ritmos institucionales y su posterior socialización, bajo la forma de educación sanitaria, puede generar una cierta cultura del uso del $\boldsymbol{S U H}$, en la que se desincentivan ciertos comportamientos considerados como inadecuados y se priorizan aquellos considerados como adecuados. Seguramente este mismo proceso habría que realizarlo en el CS de tal forma que se pueda generar una cierta cultura del uso del CS. Lo cierto es que la cada vez mayor complejidad de la asistencia sanitaria impone a las instituciones de salud un proceso activo, tanto de formalización de nuevos marcos de cultura institucional, particularmente en relación el uso de los servicios, como de socialización a través de la figura de la educación sanitaria. Esto, a su vez, puede contribuir a una mejor adecuación de las demandas y las expectativas de los usuarios a los nuevos escenarios de atención sanitaria. 


\section{BIBLIOGRAFÍA}

AfIlalo, M., et al. (1995): Emergency department use and misuse. The Journal of Emergency Medicine 13(2): 259-264.

Afilalo, J., Marinovich, A., Afilalo, M., Colacone, A., Le'Ger, R., Unger, B, Gigue`re, C. (2004): Nonurgent Emergency Department Patient Characteristics and Barriers to Primary Care, Acad Emerg Med. 11 (12): $1302-1310$.

Alonso, M., Hernández, R., Del Busto Prado, F., Cueto, A., (1993): Utilización de un Servicio de Urgencias Hospitalario, Rev San Hig Púb 67(1): 3945-45

American College of EMergency Physicians (2008): Emergency Department Crowding: High-Impact Solutions. ACEP Task Force Report on Boarding. Internet:

http://www.acep.org/workarea/downloadasset.aspx?id=37960 (última consulta el 11 de diciembre de 2009).

AminZADEH, F., DAlZIEL, W., (2002): Older adults in the emergency department: A systematic review of patterns of use, adverse outcomes, and effectiveness of interventions. Ann Emerg Med 39(3): 238-247.

ANDERSEN, R. (1995): Revisiting the Behavioral Model and Access to Medical Care: Does it Matter? J Health Social Behavior 36(1): 1-10.

Andersen, R., Newman, F. (2005): Societal and Individual Determinants of Medical Care Utilization in the United States, The Milbank Quarterly 83(4): 1-28.

AndRen, K. G., Rosenqvist, U. (1985): Heavy Users of an Emergency Department: Psychosocial and Medical Characteristics, Other Health Care Contacts, and the Effect of a Hospital Social Worker Intervention. Social Science and Medicine 21(7): 761-770.

ANDRÉn, K., Rosenqvist, U. (1987): Heavy Users of an Emergency Department: A Two Year Follow-up Study. Social Science and Medicine 25(7):825-831.

ArAnAz, J., et al. (2006): ¿Por qué los pacientes utilizan los servicios de urgencias hospitalarios por iniciativa propia?, Gaceta Sanitaria, 20(4):311-5.

Arkun, A., Briggs, W., Patel, S., Datillo, P., Bove, J., Birkhahn, R. (2010): Emergency department crowding: factors influencing flow. West J Emerg Med. 11(1):10-5.

Asplin, B., Magid, D., Rhodes, K., Solberg, L., Lurie, N., Camargo, C., (2003): A Conceptual Model of Emergency Department Crowding, Ann Emerg Med 42(2):173-180.

Ayuntamiento de Parla (2010): Perfil Demográfico de la Población. Departamento de Estadística. Datos extraídos del Padrón Municipal de habitantes a 1 de enero de 2010.

Backman, A., Blomqvist, P. Lagerlund, M., Carlsson-Holm, E., AdAmi1, J. (2008): Characteristics of nonurgent patients: Cross-sectional study of emergency department and primary care patients. Scandinavian Journal of Primary Health Care 26(3): 181-187.

Bernstein, S., et al. (2009): The Effect of Emergency Department Crowding on Clinically Oriented Outcomes, Acad Emerg Medicine 16(1):1-10.

Béland, F., Lemay, A., Boucher, M. (1998): Patterns of visits to hospital emergency rooms. Social Science and Medicine 47(2): 165-179.

Berry, A., Brousseau. D., Brotanek; J., Tomany-Korman, S., Flores, G. (2008): Why Do Parents Bring Children to the Emergency Department for Nonurgent Condition? A qualitative study. Ambulatory Pediatrics; 8(6): 360-367.

Bianco, A. Pileggi, C., Angelillo, I. (2003): Non-urgent visits to a hospital emergency department in Italy, Public Health 117(4) 250-255.

Bianco, A., Foresta, M., Greco, M., Teti, V., Angelillo, I. (2002): Appropriate and inappropriate use of day-hospital care in Italy. Public Health (2002) 116(6): 368-373.

Boushy, D., DuBINSKY, I. (1999): Primary care physician and patient factors that result in Patients seeking emergency care in a hospital setting: The patient's perspective, The Journal of Emergency Medicine 17(3): 405-412.

BRIM, C. (2008): A descriptive analysis of the non-urgent use of emergency departments. Nurse Researcher. 15(3): $72-88$

Bragulat, E., et al. (2003): Saturación del servicio de urgencias: factores asociados y cuantificación, Med Clín 121(5):167-172. 
Burnett, M., Grover, S. (1996): Use of the Emergency Department for No urgent Care During Regular Business Hours, Can Med Assoc J 154 (9) 1345-1351.

CARret, M., FAssa, A., KaWACHI, A. (2007): Demand for emergency health service: factors associated with inappropriate use. BMC Health Services Research. 7:131.

CARret, M., FASSA, A., Domingues, M. (2009): Inappropriate use of emergency services: a systematic review of prevalence and associated factors, Cad Saude Publica 25(1):7-28.

Chan, B., Ovens, H. (2002): Frequent users of emergency departments. Do they also use family physicians' services? Canadian Family Physician 48: 1654-60.

Derlet, R., Richards, J. (2000): Overcrowding in the Nation's Emergency Departments: Complex Causes and Disturbing Effects, Annals of Emergency Medicine 35(1): 63-68.

Derlet, R., Richards, J., Kravitz, R. (2001): Frequent Overcrowding in U.S. Emergency Departments, Acad Emerg Med. 8(2):151-155.

Durand, A., Gentile, S., Devictor, B., Palazzolo, S., Vignally, P., Gerbeaux, P., Sambuc, R. (2010): ED patients: how nonurgent are they? Systematic review of the emergency medicine literature. Am J Emerg Med. [Epub ahead of print].

Driscoll, P., Vincent, C., Wilkinson, M (1987): The use of the accident and emergency department, Arch Emerg Med 4(2): 77-82.

Europa Press (2002): Miles de vecinos de Parla exigen en la Puerta del Sol un hospital para su municipio. En El Mundo [en línea], lunes, 10 de Junio. Internet: http://www.elmundo.es/elmundo/2002/06/09/madrid/ 1023606577.html (última consulta 26 de noviembre de 2010).

FAtovich, D., HiRsch, R. (2003): Entry overload, emergency department overcrowding, and ambulance bypass, Emerg Med $J$ 20(5):406-409.

FERnANDES, Ch., et al (2005): Five-Level Triage: A Report from the ACEP/ENA Five-Level: Triage Task Force, Journal Of Emergency Nursing 3(1): 39-50.

FIELD, S., LANTZ, F. (2006): Emergency department use by CTAS Levels IV and V patients. CJEM 8(5):317-322.

Gentile, S., Vignally, P., Durand, A., Gainotti, S., Sambuc, R. Gerbeaux, P. (2010): Nonurgent patients in the emergency department? A French formula to prevent misuse. BMC Health Services Research, 10:66.

GILl, J. (1999): Use of Hospital Emergency Departments for Nonurgent Care: A Persistent Problem with No Easy Solutions, The American Journal of Managed Care 5(12): 1565-1568.

GILL, J., RiLEY, A. (1996): Nonurgent use of hospital emergency department: urgency fron the patient's perspective, Journal of Family Practice, 42(5):491-6.

Gill, J., Mainous, A., Nsereko, M. (2000): The effect of continuity of care on emergency department use. Arch Fam Med 9(4):333-8.

GoMEZ, J. (2006): Urgencias, gravedad y complejidad: un constructo teórico de la urgencias basado en el triaje estructurado, Emergencia 18 (3): 156-164

Grumbach, K, Keane, D., Bindman, A. (1993): Primary Care and Public Emergency Department Overcrowding, American Journal of Public Health 83(3):372-378.

Guttman, N., Zimmerman, D., Schaub, M. (2003): The Many Faces of Access: Reasons for Medically Nonurgent, Emergency Department Visits Journal of Health Politics, Policy and Law 28(6): 1089-1020.

Han, A., Ospina, M., Blitz, S., Strome, T., Rowe, B. (2007): Patients presenting to the emergency department: the use of other health care services and reasons for presentation Can J Emerg Med 9(6):428-34.

Hansagi, H., Olsson, M., Sjoberg, S., Tomson, Y., Goransson, S. (2001): Frequent Use of the Hospital Emergency Department Is Indicative of High Use of Other Health Care Services, Annals of Emergency Medicine 37(6): 561-567.

Hoot, N., Aronsky, D. (2008): Systematic Review of Emergency Department Crowding: Causes, Effects, and Solutions. Ann Emerg Med 52(2):126-136.

Hospital de Parla, (2009): Actividad total 2008.

Hospital de Parla, (2010): Actividad total 2009.

Howard, M., et al. (2005): Patients' Perspective on Choosing the Department for Nonurgent Medical Care: A Qualitative Study Exploring One Reason for Overcrowding, Emergency Journal of Emergency Nursing 31(5): 429-435. 
Hwang, U., Concato, J. (2004): Care in the Emergency Department: How Crowded Is Overcrowded? Acad Emerg Med 11(10): 1097-1101

Instituto DE Estadística (2007): Indicador de Renta Disponible Bruta Municipal por zonas estadísticas y por municipios. Internet: http://www.madrid.org/iestadis/fijas/estructu/economicas/datosimpositivos/ iirfdmb00.htm (última consulta 29 de octubre de 2009).

InSTITUTO DE ESTADÍSTICA, ALMUDENA, Banco de datos municipal y zonal. Internet: http://www.madrid.org/desvan/ almudena/almudena.html (última consulta 29 de octubre de 2009).

Instituto DE EstadísticA, Ficha Municipal: Parla. Internet: http://www.madrid.org/ICMdownload/ ficha 7iYe5e.pdf (última consulta 29 de octubre de 2009).

Instituto Nacional de Estadística (2009a): Defunciones según la Causa de Muerte 2007. Internet: http://www.ine.es/jaxi/menu.do?type=pcaxis\&path=/t15/p417/a2007/\&file=pcaxis (última consulta 3 de diciembre de 2009)

Instituto Nacional de Estadística (2009b): Establecimientos Sanitarios con Régimen de Internado. Serie 1997-2006. Internet: $\mathrm{http} / / \mathrm{www}$. ine.es/jaxi/menu.do?type $=$ pcaxis\&path $=\% 2 \mathrm{Ft} 15 / \mathrm{p} 123 \&$ file $=$ inebase $\& \mathrm{~L}=0$ (última consulta 26 de diciembre de 2009).

Instituto Nacional de Estadística (2009c): Encuesta Nacional de Salud, 2003, 2006. Internet: http://www.ine.es $/$ jaxi/menu.do?L $=0 \&$ type $=$ pcaxis\&path $=\% 2 \mathrm{Ft} 15 / \mathrm{p} 419 \&$ file $=$ inebase $($ última consulta 6 de diciembre de 2009).

Instituto Nacional de Estadística (2009d): Cifras de población. Series Históricas de Población. Internet: http://www.ine.es/jaxi/menu.do?type $=$ pcaxis \&path $=\% 2 \mathrm{Ft} 20 \% 2 \mathrm{Fe} 245 \% 2 \mathrm{Fp} 05 \&$ file $=$ inebase $\& \mathrm{~L}=($ última consulta 5 de diciembre de 2009)

Ionescu-Ittu, R., McCusker, J., Ciampi, A., Vadeboncoeur, A., Roberge, D., Larouche, D., Verdon, J, PINEAULT, R. (2007): Continuity of primary care and emergency department utilization among elderly people. CMAJ, 177(11):1362-8.

Kierzek, G., RaC, V., Pourriat, J. (2010): Emergency Department Workload Increase: Dependence on Primary Care? J Emerg Med 38(3): 279-286.

Koziol-McLain, J., Price, D., Weiss, B., Quinn, A., Honigman, B. (2000): Seeking care for nonurgent medL cal conditions in the emergency department: Through the eyes of the patient, Journal of Emergency Nursing 26(6): 554-563.

KRUG, S. (1999): Access and use of emergency service: inappropriate use versus unmet need, Clin Ped Emerg Med 1(1): 35-44.

Land, F., Boucher, L., Boucher, A. (1998): Patterns o Visits t Hospital-Based Emergency Rooms, Social Science and Medicine 47(2): 165-179.

Lang, T., Davido, A., Diakite, B., Agay, E., Viel, JF., Flicoteaux, B. (1996): Non-urgent care in the hospital medical emergency department in France: how much and which health needs does it reflect? Epidemiol Community Health 50(4):456-462.

Lang, T., Davido, A., Diakité, B., Agay, E., Viel, J. (1997): Using the hospital emergency department as a regular source of care, European Journal of Epidemiology 13(2): 223-228.

Lee, A., Hazlett, C., Chow, S, Lau, F., Kam, Ch., Wong, P., Wong, T. (2003): How to minimize inappropriate utilization of Accident and Emergency Departments: improve the validity of classifying the general practice cases amongst the A\&E attendees, Health Policy 66 (2): 159-168.

Ley General de SANIDAd, Segunda Edición, Tercera reimpresión, Madrid: Tecnos 1997.

LIGGINS, K., (1993): Inappropriate attendance at accident and emergency department: a literature review, $J A d v$ Nursing, 18 (7): 1141-1145.

Lee, A., Lau, F., Hazlett, C., Kam, Ch., Wong, P., Wong, T., Chow, C. (2000): Factors associated with nonurgent utilization of Accident and Emergency services: a case-control study in Hong Kong, Social Science and Medicine 51(7): 1075-1085.

Lowe, R., Bindman, A. (1997): Judging Who Needs Emergency Department Care: a Prerequisite for PolicyMaking, Am J Emerg Med 15 (2): 133-136.

Lowthian, J., Curtis,A., Cameron, P., Stoelwinder, J., Cooke, M., McNeil, J. (2010): Systematic review of trends in emergency department attendances: an Australian perspective, Emerg Med $J$ [On line]: 
http://emj.bmj.com/content/early/2010/10/20/emj.2010.099226.full?sid=858061ec-90b4-4754-b48f42431db9a73d (última consulta 27 de noviembre de 2010).

Malone, R. (1995): Heavy Users of Emergency Services: Social Construction of a Policy Problem, Social Science and Medicine 40(4): 469-477.

MÁRQUEZ, J., et al. (2007): Diferencias en los motivos de consulta entre pacientes que acuden derivados y por iniciativa propia a un Servicio de Cuidados Críticos y Urgencias de un hospital general. Emergencias 19(2): 70-76.

Ministerio de Sanidad y Política Social (2009): Barómetro Sanitario 1995-2008. Instituto de Información Sanitaria. Internet: http://www.msc.es/estadEstudios/estadisticas/inforRecopilaciones/barometro/home.htm (última consulta 7 de diciembre de 2009).

Moskop, J., Sklar, D., Geiderman, J., Schears, R., Bookman, K. (2009a): Emergency Department Crowding, Part 1-Concept, Causes, and Moral Consequences. Annals of Emergency Medicine 53(5): 605-611.

Moskop, J., Sklar, D., Geiderman, J., Schears, R., Bookman, K. (2009b): Emergency Department Crowding, Part 2-Barriers to Reform and Strategies to Overcome Them, Annals of Emergency Medicine 53(5): $612-61$.

MuRPHY, A. (1998): "Inappropriate" attenders at accident and emergency department I: definition, incidence and reasons for attendance, Family Practice 15(1): 23-32.

Murray, M., Bullard, M, Grafstein, E. (2004): Revisions to the Canadian Emergency Department Triage and Acuity Scale Implementation Guidelines, Can J Emerg Med 6(6):421-7

National Health System (2009): Hospital Episode Statistics: A\&E Attendances in England (Experimental Statistics), 2007-08. Internet: http://www.ic.nhs.uk/statistics-and-data-collections/hospital-care/accidentand-emergency-hospital-episode-statistics-hes/accident-and-emergency-attendances-in-england-experimental-statistics-2007-08 (última consulta 10 de diciembre de 2009)

NAIRn, W., Marshal, R., Swann, G. (2004): The patient experience in emergency departments: a review of the literature. Accid Emerg Nurs. 12(3):159-65.

Northington, W., Brice, J., Zou, B. (2005): Use of an emergency department by nonurgent patients American, Journal of Emergency Medicine 23(2): 131-137

OBSERVATORIO DE INMIGRACIÓN- DENTRO DE ESTUDIOS Y DATOS (2009): Informe de la población extranjera empadronada en la Comunidad de Madrid a Enero de 2009. Consejería de Inmigración y de Cooperación.

O'BRIEN, G., et al (1996): "Inappropriate Emergency Department Use: A Comparison of three Methodologies for Identification, Acad Emerg Med 3(3): 252-257.

Ochoa J, Ramalle-Gómara E, Villar A, Ruiz JI, Bragado L, Gimeno C. (2000): Visitas inapropiadas al servicio de urgencias de un hospital general. Medicina Clinica (Barc), 115(10):377-8.

Oktay, C., Cete, Y., Eray, O., Pekdemir, M., Gunerli, A. (2003): Appropriateness of Emergency Department Visits in a Turkish University Hospital. Croat Med J. 44(5): 585-591.

Olsson, M., Hansagi, H., (2001): Repeated use of the emergency department: qualitative study of the patient's perspective. Emerg Med J. 18(6): 430-434.

Oterino de la Fuente, D., Baños, J., Fernandez, V., Rodriguez A. (2006): Does better access to primary care reduce utilization of hospital accident and emergency departments? A time-series analysis. European Journal of Public Health, 17(2):186-192.

PASARIN, M., et al. (2006): Razones para acudir a los servicios de urgencias hospitalarios: La población opina, Gac Sanit 20(2): 91-99.

PAdgetT, D., Brodsky, B. (1992): Psychosocial Factors Influencing Nonurgent Use of the Emergency Room: A Review of the Literature and Recommendations for Research and Improved Service Delivery. Social Science and Medicine 35(9):1189-1197.

Pereira, S., et al. (2001): Appropriateness of Emergency Department Visits in a Portuguese University Hospital. Annals of Emergency Medicine 37(6): 580-586.

PÉREZ, R. (2007): Mil razones para acudir a urgencias. La asistencia integral del hospital compensa la espera y la posible falta de beneficio clínico, El País, febrero 20. Internet: http://www.elpais.com/articulo/ salud/Mil/razones/acudir/urgencias/elpepusocsal/20070320elpepisal_1/Tes (última consulta 7 de diciembres de 2009). 
Pescosolido, B. (1992): Beyond Rational Choice: The Social Dynamics of How People Seek Help, The American Journal of Sociology, 97(4): 1096-1138.

Phillips, K., Morrison, K., ANDERSEn, R., AdAY, L: (1998): Understanding the Context of Healthcare Utilization: Assessing Environmental and Provider-Related Variables in the Behavioral Model of Utilization, Health Services Research 33(3): 571-596.

PitTs, S., NisKa, R., Xu, J., BuRT, C. (2008): National Hospital Ambulatory Medical Care Survey: 2006 Emergency Department Summary, National Health Statistics Reports Number 7: 1-40.

Puigblanqué, E., Noheras, Marc., Nogueras, M., Perapoch, N. (2008): El triaje en el servicio de urgencias, Enferm Clin 18(1):3-4.

Ragin, D., Hwang, U., Cydulka, R., Holson, D., Haley, Ll Jr., Richards, C., Becker, B., Richardson, L. (2005): Reasons for using the emergency department: results of the EMPATH Study, Acad Emerg Med 12(12):1158-66.

Rajpar, S., Smith, M., CoOKe, M. (2000): Study of choice between accident and emergency departments and general practice centres for out of hours primary care problems J Accid Emerg Med 200017 (1):18-21.

Richardson, L., Hwang, U. (2001a): Access to Care: A Review of the Emergency Medicine Literature, Acad Emerg Medicine 8 (11):1030-1036.

RichardSON, L., HwANG, U., (2001b): America's Health Care Safety Net: Intact or Unraveling? Acad Emerg Medicine 8(11):1056-1063.

Richardson, L., Asplin, B., Lowe, R. (2002): Crowding as a health policy issue: past development, future directions. Ann Emerg Med 40(4):388-93.

Rubin, M., Bonnin, M. (1995): Utilization of the Emergency Department by Patients with Minor Complaints, The Journal of Emergency Medicine 13(6): 839-842.

Rust, G., Ye, J., Baltrus, P., Daniels, E., Adesunloye, B., Fryer, G. (2008): Practical Barriers to Timely Primary Care Access Impact on Adult Use of Emergency Department Services, Arch Intern Med 168(15):705710.

Salvi, F., Morichi, V., Grilli, A., Giorgi, R., De Tommaso, G., Dessì-Fulgheri, P. (2007): The elderly in the emergency department: a critical review of problems and Solutions, Internal and Emergency Medicine 2(4): 292-301.

Samaras, N., Chevalley, T., Samaras, D., Gold. G. (2010): Older patients in the emergency department: a review. Ann Emerg Med. 56(3):261-9.

SÁNCHEZ, J., BuEno, A. (2005): Factores asociados al uso inadecuado de un servicio de urgencias hospitalario. Emergencias 17(3):138-144.

SÁnChEZ, J., CAVAnILlas, A. (2005): Factores asociados al uso inadecuado de un servicio de urgencias hospitalario, Emergencias. 17(3): 138-144.

Sánchez, J., Cavanillas, A., Delgado, A., Muñoz, H., Jiménez, J., Luna del Castillo. J (2005): Atención Primaria asignada y uso de la urgencia hospitalaria, Emergencias. 17(6): 251-259.

Sánchez, M., Miró, O., Coll-Vinent, B., Bragulat, E., Espinosa, V., Gómez-Angelats, E., Jiménez, S., Queralt, C., Hernández-Rodríguez, J., Alonso J., Millá, J. (2003): Saturación del servicio de urgencias: factores asociados y cuantificación, Med Clin (Barc) 121(5):167-72.

SÁNCHEZ, M., OrTiZ, C. (2007): La realidad de las urgencias hospitalarias, El Mundo, martes, 13 de febrero, número: 6267. Internet:

http://www.elmundo.es/papel/2007/02/13/madrid/2083835.html (última consulta el 7 de diciembre de 2009)

SANDERS, J. (2000): A review of health professional attitudes and patient perceptions on 'inappropriate' accident and emergency attendances. The implications for current minor injury service provision in England and Wales, Journal of Advanced Nursing, 31(5): 1097-1105.

SARVER, J., CydUlKa, R., BAKER, W. (2002): Usual source of care and nonurgent emergency department use. Acad Emerg Med 9(9):916-23.

Schneider, S., Zwemer, F., Doniger, A., Dick, R., Czapranski, T., Davis, E., (2001): Rochester, New York: A Decade of Emergency Department Overcrowding, Acad Emerg Medicine 8(11):1044-1050.

Schneider, S., Gallery, M., Schafermeyer, R., Zwemer, F. (2003): Emergency Department Crowding: A Point in Time, Ann Emerg Med. 4(2):167-172. 
Schull, MD, M., Slaughter, P., Redelmeier, D. (2002): Urban emergency department overcrowding: defining the problem and eliminating misconceptions, CJEM 4 (2): 76-83.

Sempere, T., Peiró, S., Sendra, P. (L), Martínez, C., LóPez, I. (1999): Validez del Protocolo de Adecuación de Urgencias Hospitalarias, Rev Esp Salud Pública 73(4): 465-479.

Sempere-Selva, T., Peiró, S., Sendra, P., Martínez C., LóPez, I. (2001): Inappropriate use of an Accident and Emergency Department: Magnitude, Associated factors, and reasons. An approach with explicit criteria, Annals of Emergency Medicine 37(6): 568-579.

SERVICIO MADRILEÑo DE SALUd (2008): Evaluación de la satisfacción de los usuarios de atención especializada: análisis por hospital.

STEAD, L., JAIN, A., DeCKER,W . (2009): Emergency department over-crowding: a global perspective, Int J Emerg Med 2(3):133-134.

Strange, G., Chen, E. (1998): Use of emergency departments by older patients: A five-year follow-up study, Acad Emerg Med 5(12): 1157-1162.

Tranquada, K., Denninghoff, K., King, M., Davis, S., Rosen, P. (2010): Emergency Department Workload Increase: Dependence on Primary Care? The Journal of Emergency Medicine 38(3): 279-285.

TRZECIAK, S., RIVERS, E. (2003): Emergency department overcrowding in the UnitedStates: an emerging threat to patient safety and public Health, Emerg Med J 20(5):402-405.

US Department of Health And Human Services (2008): National Health Statistics Reports: National Hospital Ambulatory Medical Care Survey: 2006 Emergency Department Summary, Number 7.

VÁzquez, B., Pardo, G., Fernández, G., Canals, M., Delgado. M., Navas, M. (2000): ¿Por qué acuden nuestros pacientes a urgencias del hospital? Aten Primaria. 25(3): 98-105.

WeISS, S., et al (2004): Estimating the Degree of Emergency Department Overcrowding in Academic Medical Centers: Results of the National ED Overcrowding Study (NEDOCS), Acad Emerg Medicine 11(1):38-50.

Wong; W., Edgar, G., Liddy, C., Vaillancourt, CH., (2009): Can after-hours family medicine clinics represent an alternative to emergency departments? Survey of ambulatory patients seeking after-hours care, Can Fam Physician 55(11):1106-7.

Zaragoza, M., Calvo, C, SaAd T., Morán, F., San José, S., Hernández, P. (2009): Evolución de la frecuentación en un servicio de urgencias hospitalario, Emergencias 21(5): 339-345; Aranaz, J., et al. (2006): ¿Por qué los pacientes utilizan los servicios de urgencias hospitalarios por iniciativa propia?, Gaceta Sanitaria, 20(4):311-5. 